\title{
Is there a renoprotective value to leukodepletion during heart valve surgery? A randomized controlled trial (ROLO)
}

Espeed Khoshbin ${ }^{1 *} \mathbb{D}$, Sally Spencer², Laurence Solomon ${ }^{3}$, Augustine Tang ${ }^{4}$, Stephen Clark', Elizabeth Stokes ${ }^{5}$, Sarah Wordsworth ${ }^{5}$, Lucy Dabner ${ }^{6}$, Julia Edwards ${ }^{6}$, Barnaby Reeves ${ }^{6}$ and Chris Rogers ${ }^{6}$

\begin{abstract}
Background: Acute Kidney Injury (AKI) adversely affects outcomes after cardiac surgery. A major mediator of AKI is the activation of leukocytes through exposure to the cardiopulmonary bypass circuit. We evaluate the use of leukodepletion filters throughout bypass to protect against post-operative AKI by removing activated leukocytes during cardiac surgery.

Methods: This is a single-centre, double-blind, randomized controlled trial comparing the use of leukodepletion versus a standard arterial filter throughout bypass. Elective adult patients undergoing heart valve surgery with or without concomitant procedures were investigated. The primary clinical outcome measured was the development of AKI according to the KDIGO criteria. Secondary measures included biomarkers of renal tubular damage (urinary Retinol Binding Protein and Kidney Injury Molecule-1), glomerular kidney injury (urinary Micro Albumin and serum Cystatin C) and urinary Neutrophil Gelatinase Associated Lipocalin, as well as the length of hospital stay and quality of life measures through EQ-5D-5L questionnaires.
\end{abstract}

Results: The ROLO trial randomized 64 participants with a rate of recruitment higher than anticipated (57\% achieved, $40 \%$ anticipated). The incidence of AKI was greater in the leukodepletion filter group (44\% versus $23 \%$, risk difference $21,95 \% \mathrm{Cl}-2$ to $44 \%)$. This clinical finding was supported by biomarker levels especially by a tendency toward glomerular insult at $48 \mathrm{~h}$, demonstrated by a raised serum Cystatin $C$ (mean difference $0.11,95 \% \mathrm{Cl} 0.00$ to $0.23, p=$ 0.068) in the leukodepleted group. There was however no clear association between the incidence or severity of AKI and length of hospital stay. On average, health related quality of life returned to pre-operative levels in both groups within 3 months of surgery.

Conclusions: Leukocyte depletion during cardiopulmonary bypass does not significantly reduce the incidence of AKI after valvular heart surgery. Other methods to ameliorate renal dysfunction after cardiac surgery need to be investigated.

* Correspondence: Espeed.Khoshbin1@nhs.net

'Department of Cardiothoracic Surgery, Freeman Hospital, High Heaton, Newcastle upon Tyne NE7 7DN, UK

Full list of author information is available at the end of the article

(C) The Author(s). 2021 Open Access This article is licensed under a Creative Commons Attribution 4.0 International License, which permits use, sharing, adaptation, distribution and reproduction in any medium or format, as long as you give appropriate credit to the original author(s) and the source, provide a link to the Creative Commons licence, and indicate if changes were made. The images or other third party material in this article are included in the article's Creative Commons. licence, unless indicated otherwise in a credit line to the material. If material is not included in the article's Creative Commons licence and your intended use is not permitted by statutory regulation or exceeds the permitted use, you will need to obtain permission directly from the copyright holder. To view a copy of this licence, visit http://creativecommons.org/licenses/by/4.0/ The Creative Commons Public Domain Dedication waiver (http://creativecommons.org/publicdomain/zero/1.0/) applies to the data made available in this article, unless otherwise stated in a credit line to the data. 
(Continued from previous page)

Trial registration: The trial was registered by the International Standard Randomized Controlled Trial Number Registry ISRCTN42121335. Registered on the 18 February 2014. The trial was run by the Bristol Clinical Trials and Evaluation Unit. This trial was financially supported by the National Institute of Health Research (Research for Patient Benefit), award ID: PB-PG-0711-25,090.

Keywords: Cardiac surgery, Heart valve, Cardiopulmonary bypass, Acute kidney injury, Leukodepletion

\section{Introduction}

Following cardiac surgery the overall incidence of acute kidney injury (AKI) as defined by RIFLE (Risk, Injury, Failure, Loss of kidney function) criteria is $23 \%[1,2]$. Patients undergoing valvular heart surgery are at a particularly higher risk of AKI [3]. Heart valve surgery is an independent risk factor for the development of AKI which has been detected in up to $40 \%$ of these patients $[4,5]$. This may be due to a relatively longer period of cardiopulmonary bypass.

AKI is associated with a twofold increase in mortality, prolonged ICU stay, chronic ill-health and a 1.6 fold increase in the financial cost of care with the risk to costs ratio escalating with the severity of AKI $[6,7]$. Therefore AKI significantly and adversely affects prognosis and health outcome after cardiac surgery $[8,9]$.

Central to the complex etiology of peri-operative renal damage is the systemic inflammatory response to cardiopulmonary bypass (CPB). The important role of leukocyte activation in $\mathrm{CPB}$-associated inflammation is universally recognized [10-13]. It has been previously hypothesized that the inherent nephrotoxic effect of CPB may be significantly reduced by incorporating a leukodepletion filter into the circuit (LG-6 LeukoGuard ${ }^{\bullet}$ Leukocyte ReductionPall Corporation) to physically remove activated leukocytes [14-17]. However the effectiveness of leukodepletion in reducing $\mathrm{CPB}$-associated renal impairment has not been fully evaluated [18].

We tested the hypothesis that leukocyte depletion during bypass would ameliorate renal injury in patients undergoing heart valve surgery as this group of patients have a higher risk of developing bypass related AKI [4]. Previous study of a low risk cohort undergoing coronary surgery alone did not demonstrate clinical benefit [15].

\section{Methods}

AKI was defined by Kidney Disease Improving Global Outcomes (KDIGO) criteria (Table 1) in patients undergoing heart valve surgery.

This single-centre, double-blind study was designed as an external feasibility trial comparing two parallel groups. Patients were randomized to receive either a leukodepletion filter or a standard arterial filter as part of the bypass circuit. The filter was used throughout the bypass run from start to finish.

The frequency of AKI, clinical and sub clinical data, health-related quality of life (HRQoL) and resource use data was investigated and collected. We assessed the sub clinical effects of leukodepletion through measurement of urinary and serum biomarkers of tubular and glomerular kidney injury. Acute injury to the renal tubules was assessed by excretion of retinol binding protein (RBP) in the urine (ELISA methods). This biomarker has previously been validated in cardiac surgical patients [19, 20]. More recent evidence suggests urinary Kidney Injury Molecule-1 (KIM-1) as a superior biomarker (ELISA method) than RBP $[20,21]$. Acute injury to the renal glomeruli was assessed by urinary micro Albumin (Alb) through turbimetric assay [22] and by serum Cystatin-C concentration, with potentially better sensitivity compared to glomerular filtration rate [23]. These biomarkers allow for early detection of renal injury before conventional clinical parameters such as blood urea and serum Creatinine, become abnormal.

Fresh urine samples were collected within strict time 'windows' for each biomarker. Urinary RBP, KIM-1 and Alb were indexed to urinary excretion of Creatinine (RBP:Cr, Alb:Cr, KIM-1:Cr) to adjust for variations in the glomerular filtration rate. Urinary Neutrophil Gelatinase Associated Lipocalin (NGAL) [24] another biomarker used in cardiac surgery to assess associated AKI

Table 1 Kidney Disease Improving Global Outcomes (KDIGO): primary outcome defined by this criterion for acute kidney injury

\begin{tabular}{lll}
\hline Stage & Serum creatinine $(\mathrm{SCr})$ criteria & Urine output criteria \\
\hline 1 & Increase $\geq 26 \mu \mathrm{mol} / \mathrm{L}$ within $48 \mathrm{~h}$ or & $<0.5 \mathrm{~mL} / \mathrm{kg} / \mathrm{hr}$. for $>6$ consecutive hrs \\
& increase $\geq 1.5$ to $1.9 \times$ reference baseline $\mathrm{SCr}$ & $<0.5 \mathrm{~mL} / \mathrm{kg} / \mathrm{hr}$. for $>12 \mathrm{~h}$ \\
3 & Increase $\geq 2$ to $2.9 \times$ reference baseline $\mathrm{SCr}$ & $<0.3 \mathrm{~mL} / \mathrm{kg} / \mathrm{hr}$. for $>24 \mathrm{~h} \mathrm{or} \mathrm{anuria} \mathrm{for} 12 \mathrm{~h}$ \\
& $\begin{array}{l}\text { Increase } \geq 3 \times \text { reference baseline } \mathrm{SCr} \text { or } \\
\text { increase } \geq 354 \mu \mathrm{mol} / \mathrm{L} \text { or } \\
\text { commenced on renal replacement therapy irrespective of stage }\end{array}$ & \\
\hline
\end{tabular}


and serum Cystatin $\mathrm{C}$ was measured as actual concentrations.

Comparative analyses of levels of biomarkers over time was carried out using mixed regression models, including main effects of group, time and the interaction of group by time. This allowed for a more precise assessment of AKI than can be achieved using the KDIGO criteria, which does not differentiate between pre-renal failure (due to volume depletion) and renal damage.

HRQoL was measured using the Minnesota Living with Heart Failure (MLHF) [25] and EQ-5D-5L questionnaires enabled the evaluation of both impact on quality of life and cost (resource use data) in a single measure $[26,27]$ up to 3 months following surgery.

The sample size was set at 108 participants (54 per group) who would allow the incidence of AKI to be estimated with the following precision $(95 \%$ confidence interval): 31 to $50 \%$ for an incidence of 40 and $5 \%$ to $17 \%$ for an incidence of $10 \%$ (Proportions as high as $40 \%$, and as low as $10 \%$, have been reported to have developed AKI following heart valve surgery). This sample size also had $95 \%$ power to detect a 1.5 fold reduction in RBP:Cr for the leukodepletion filter group at the 1\% level of statistical significance [15], allowing for $10 \%$ dropout. The 1.5 fold reduction is lower than that observed in a previous study for both RBP:Cr and Alb:Cr [15], but was considered clinically relevant and appropriate as the previous study may have been at risk of bias.

Inclusion and exclusion criteria are illustrated in Table 2.

Eligible patients were randomized to either the leukodepletion filter (LG-6) or the standard filter group by the perfusionist using a secure password-protected webbased interface. The process took place immediately prior to setting up the appropriate filter in order to minimize dropout between randomization and surgery.

Table 2 Inclusion and exclusion criteria

\section{Inclusion criteria}

Adults aged 18-89years having single or multiple heart valve repair or replacement as a first time or redo operation as an elective or urgent procedure (i.e. non-emergency procedure), who are able to give informed consent.

Patients with or without concomitant procedures. Concomitant procedures may include but are not restricted to: coronary artery bypass graft (CABG), ascending aortic and/or root replacement, and ablation for atrial fibrillation.

Baseline urea and creatinine levels are within normal range, defined as follows: urea 2.5 to $7.8 \mathrm{mmol} / \mathrm{L}$; creatinine 45 to $90 \mu \mathrm{mol} / \mathrm{L}$ for women and 60 to $110 \mu \mathrm{mol} / \mathrm{L}$ for men.

\section{Exclusion criteria}

Baseline eGFR< $30 \mathrm{mls} / \mathrm{min} / 1.73 \mathrm{~m} 2$

Patients on renal replacement therapy

Planned deep hypothermic circulatory arrest with cardiopulmonary bypass switched off
Randomization was stratified by pre-operative risk of AKI, estimated using the Cleveland CSA-AKI Severity Score [16] (four strata, score $<3,3$ to 5,6 to $8,>8$ ). Computer generated random allocations were blocked using various block sizes. The sequence was prepared in advance by a statistician independent of the study team.

Participants, surgeons and research nurses were all blinded to the treatment allocation, only the perfusionist was aware of the filter used [28, 29].

Standard adult extracorporeal tubing set was used as per routine practice in conjunction with a D903 Avant membrane oxygenator (Sorin Biomedica, Gloucester, UK). In the control group, a standard $40-\mu \mathrm{m}$ arterial line filter was incorporated into the extracorporeal circuit downstream from the pump. This was substituted with an LG6 leukocyte-depleting filter in the study group. The circuit was primed with $1500-2000 \mathrm{~mL}$ Plasmalyte 148 solution, $1 \mathrm{~g}$ magnesium sulphate and $5000 \mathrm{IU}$ sodium heparin. An S3 Revolution centrifugal pump (Stöckert Instrumente $\mathrm{GmbH}$, Munich, Germany) delivered non-pulsatile flow maintained at $2.4 \mathrm{~L} / \mathrm{min} / \mathrm{m}^{2}$. A target mean perfusion pressure of $65 \mathrm{mmHg}$ was maintained with appropriate use of vasoactive agents. With an activated clotting time of more than $480 \mathrm{~s}, \mathrm{CPB}$ was established with mild hypothermia (lowest core temperature, $32{ }^{\circ} \mathrm{C}$ ) and alpha-stat management of acidbase status during cooling and re-warming. The filter wacovered and positioned below eye level so that it was not visible to the surgeon so to avoid un-blinding.

Adverse events (AE) were defined as any unfavorable and unintended occurrence including an abnormal laboratory finding, symptom or disease associated with the intervention or procedure, regardless of whether it is considered related to the intervention or procedure that occurs during the course of the study. Any untoward medical occurrence that resulted in death, was life threatening, required prolongation of hospitalization, resulted in persistent or significant disability/incapacity was recorded as a serious adverse event.

Analyses were based on a pre-specified statistical plan and performed on an intention-to-treat (ITT) basis. Continuous data were summarized using mean and standard deviation (or median and interquartile range if distributions were skewed) and categorical data as numbers and percentages. Continuous longitudinal outcomes were compared using linear mixed effects models. Model fit was assessed via standard methods and if inadequate then transformations or alternative analysis methods were sought. All analyses used the standard filter group as the reference group and adjusted for Cleveland AKI Severity Score (stratification factor). Outcomes were reported as proportions or effect sizes as appropriate with 95\% confidence intervals (CI) and likelihood ratio tests were used to determine statistical significance. 
Biomarker concentrations below the limit of detection were imputed using the lowest detectable concentration. All analyses were performed in Stata version 14.0 (StataCorp LP, College Station, Tex) or SAS version 9.3 (SAS Institute Inc., Cary, NC). The trial was reported according to the CONSORT reporting guidelines.

\section{Results}

Three hundred ninety-four patients were potentially eligible for the trial. one hundred forty-five patients were screened and met all the basic eligibility criteria (37, 95\% CI 32 to $42 \%$ ). Eighty-two agreed to take part in the trial (57, 95\% CI 48 to $65 \%$ ). Eight patients were found to be ineligible after consent and 10 were withdrawn. Reasons for withdrawal at this stage included surgeon preference $(n=4)$, clinical condition of the patient $(n=2)$ and logistical reasons $(n=5)$, leaving 63 randomized participants, 31 to standard perfusion and 33 to receive a leukodepletion filters (Fig. 1).

There was one treatment crossover from the leukodepletion filter to the standard filter, as the pump flow was considered too high for the leukodepletion LG-6 filter.
This was the only case where un-blinding occurred. One patient did not receive either intervention as the operation was abandoned due to an intra operative finding of a porcelain aorta.

Patient demographics are illustrated in Table 3.

The median age of participants was 72.6 years (interquartile range (IQR) 66.1 to 77.7 ) and 39/63 (62\%) were male. The median additive European System for Cardiac Operative Risk Evaluation score (EuroSCORE) was 6.0 (IQR, 4.0 to 7.0) and the median Cleveland AKI severity score was 2.0 (IQR, 1.0 to 3.0). Overall, characteristics were well balanced across the treatment groups. The groups were also equivalent in terms of preoperative co morbidities, ventricular function and the extent of coronary artery disease based on pre-operative angiogram.

The operative data are demonstrated in Table 4. Isolated valve operations accounted for 33/62 (53\%) procedures; the remainder included other procedures, mainly valve and coronary artery bypass graft (CABG) (21/62; $34 \%)$. The majority of patients had an aortic valve replacement $(47 / 62 ; 76 \%)$. The peri-operative anesthetic management was standardized and bypass data was

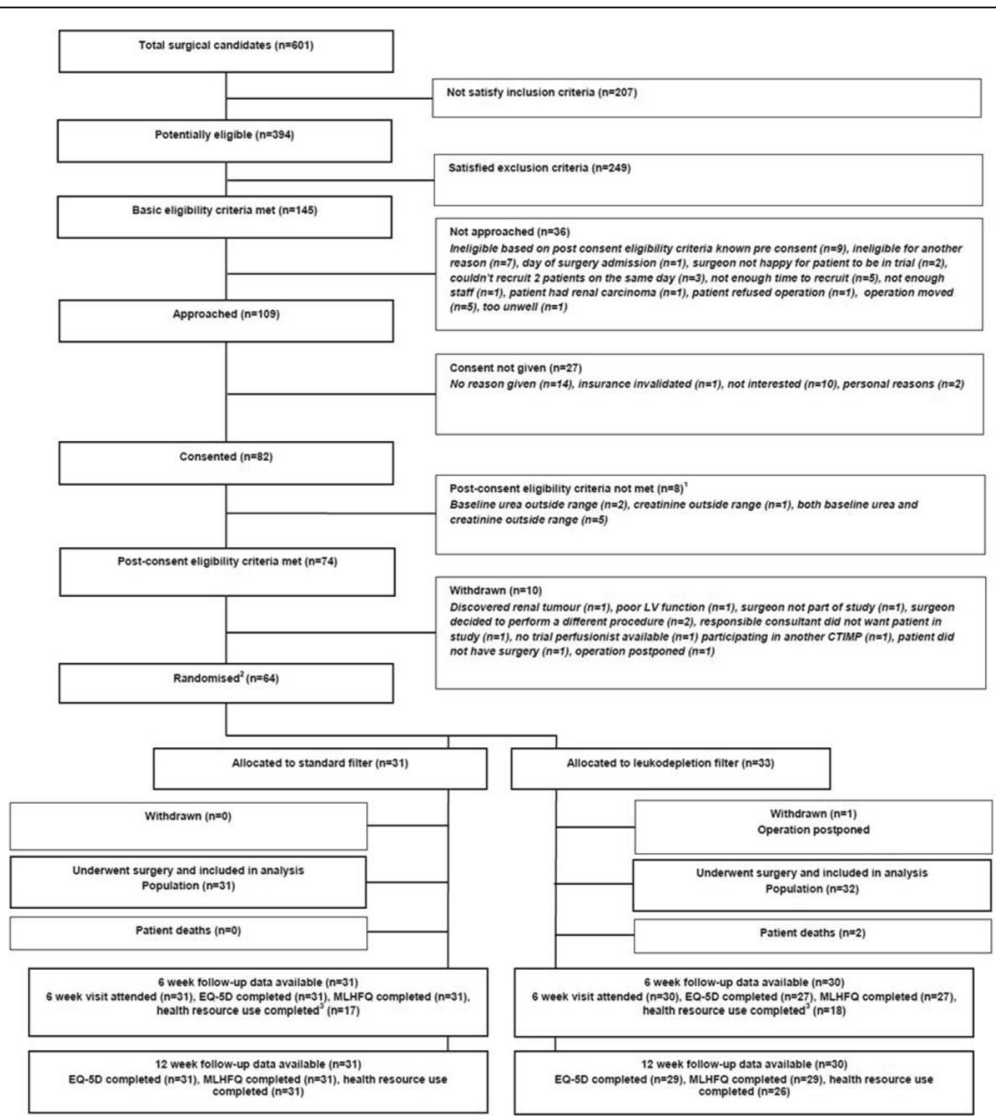

Fig. 1 Flow of participants. Notes ${ }^{\prime}$ : An additional 9th patient was found ineligible based on post-consent criteria but was included in the study based on repeat bloods taken 2: An additional 65th patient was randomized in error. As this patient did not consent and no further data was collected, this patient has not been included here. ${ }^{3}: 6$ week health resource use questionnaire brought in after trial started 
Table 3 Participant characteristics: overall, characteristics were generally well balanced across the treatment groups

\begin{tabular}{|c|c|c|c|c|c|c|c|}
\hline & & \multicolumn{2}{|c|}{$\begin{array}{l}\text { Randomized to leukodepletion } \\
\text { filter }(n=32)\end{array}$} & \multicolumn{2}{|c|}{$\begin{array}{l}\text { Randomized to standard } \\
\text { Filter }(n=31)\end{array}$} & \multicolumn{2}{|c|}{$\begin{array}{l}\text { Overall } \\
(n=63)\end{array}$} \\
\hline & & $\mathrm{n}$ & $\%$ & $n$ & $\%$ & $n$ & $\%$ \\
\hline Age (years) & Median (IQR) & 73.5 & $(69.6,77.9)$ & 72.0 & $(61.5,76.9)$ & 72.6 & $(66.1,77.7)$ \\
\hline Male & & $21 / 32$ & $66 \%$ & $18 / 31$ & $58 \%$ & $39 / 63$ & $62 \%$ \\
\hline Black ethnicity & & $2 / 32$ & $6 \%$ & $0 / 31$ & $0 \%$ & $2 / 63$ & $3 \%$ \\
\hline BMl & Median (IQR) & 27.0 & $(24.4,32.3)$ & 29.7 & $(26.0,34.6)$ & 27.8 & $(25.5,33.3)$ \\
\hline EurOSCORE & Median (IQR) & 6.0 & $(5.0,7.0)$ & 6.0 & $(3.0,7.0)$ & 6.0 & $(4.0,7.0)$ \\
\hline Cleveland CSA-AKI score & Median (IQR) & 2.0 & $(1.0,3.0)$ & 2.0 & $(2.0,3.0)$ & 2.0 & $(1.0,3.0)$ \\
\hline \multicolumn{8}{|l|}{ ECHO/ANGIOGRAM REPORT } \\
\hline \multirow[t]{2}{*}{ LV function } & Good (> 50\%) & $28 / 32$ & $88 \%$ & $26 / 31$ & $84 \%$ & $54 / 63$ & $86 \%$ \\
\hline & Moderate (30-50\%) & $4 / 32$ & $13 \%$ & $5 / 31$ & $16 \%$ & $9 / 63$ & $14 \%$ \\
\hline \multirow[t]{4}{*}{ Extent of coronary disease } & None/not investigated & $22 / 32$ & $69 \%$ & $20 / 31$ & $64 \%$ & $42 / 63$ & $66 \%$ \\
\hline & Single & $2 / 32$ & $6 \%$ & $5 / 31$ & $16 \%$ & $7 / 63$ & $11 \%$ \\
\hline & Double & $3 / 32$ & $9 \%$ & $4 / 31$ & $13 \%$ & $7 / 63$ & $11 \%$ \\
\hline & Triple & $5 / 32$ & $16 \%$ & $2 / 31$ & $6 \%$ & $7 / 63$ & $11 \%$ \\
\hline$>50 \%$ disease in left main stem & & $2 / 32$ & $6 \%$ & $2 / 31$ & $6 \%$ & $4 / 63$ & $6 \%$ \\
\hline
\end{tabular}

compatible. The median (IQR) total duration of $\mathrm{CPB}$ in minutes for the LG-6 group was $119.0(97.0,151.0)$ versus $124.0(107.0,141.0)$ for the standard filter group.

Study outcomes are summarized in Table 5.

The incidence of post-operative AKI was reported in 21/ 63 (33\%) participants (95\% CI 22 to 46\%). The incidence was higher in the leukodepletion filter group (14/32; $44 \%$ versus $7 / 31$; $23 \%$, risk difference $21,95 \%$ CI -2 to $44 \%$ ).

Most patients developed stage 1 AKI (13/20; 65\%), whereas only $10 \%$ were classified with stage 3 AKI. One patient in the leukodepletion filter group required haemodialysis. Diuretic use and fluid balance over the first 5 days were similar in the two groups. Only four patients were not given diuretics during this time.

There was no clear association between the incidence, severity of AKI and the secondary outcome of length of stay observed between the two groups (Table 5).

Hospital stay by highest AKI stage is illustrated in Fig. 2. The median length of stay in intensive care was $47.3 \mathrm{~h}$ in the leukodepletion filter group and $48.5 \mathrm{~h}$ in the standard group. The median overall post-operative hospital stay was 7.7 days and 8.8 days respectively. Two

Table 4 Operative details: the bypass data were compatible

\begin{tabular}{|c|c|c|c|c|c|c|c|}
\hline \multirow[t]{2}{*}{ Operation detail } & & \multicolumn{2}{|c|}{ Randomized to leukodepletion filter $(n=32)$} & \multicolumn{2}{|c|}{$\begin{array}{l}\text { Randomized to standard } \\
\text { filter }(n=31)\end{array}$} & \multicolumn{2}{|c|}{$\begin{array}{l}\text { Overall } \\
(n=63)\end{array}$} \\
\hline & & $\mathrm{n}$ & $\%$ & $\mathrm{n}$ & $\%$ & $\mathrm{n}$ & $\%$ \\
\hline \multirow[t]{3}{*}{ Type of surgery } & Valve & $15 / 31^{\mathrm{a}}$ & $48 \%$ & $18 / 31$ & $58 \%$ & $33 / 62$ & $53 \%$ \\
\hline & CABG and valve & $11 / 31^{a}$ & $35 \%$ & $10 / 31$ & $32 \%$ & $21 / 62$ & $34 \%$ \\
\hline & Valve and other & $5 / 31^{\mathrm{a}}$ & $16 \%$ & $3 / 31$ & $10 \%$ & $8 / 62$ & $13 \%$ \\
\hline Valve replacement & & $26 / 31^{a}$ & $84 \%$ & $25 / 31$ & $81 \%$ & $51 / 62$ & $82 \%$ \\
\hline Valve repair & & $8 / 31^{a}$ & $26 \%$ & $7 / 31$ & $23 \%$ & $15 / 62$ & $24 \%$ \\
\hline \multirow[t]{2}{*}{ Valve Replacement Details } & Aortic & $23 / 26$ & $88 \%$ & $24 / 25$ & $96 \%$ & $47 / 51$ & $92 \%$ \\
\hline & Mitral & $3 / 26$ & $12 \%$ & $1 / 25$ & $4 \%$ & $4 / 51$ & $8 \%$ \\
\hline \multirow[t]{3}{*}{ Valve Repair Details } & Mitral & $4 / 8$ & $50 \%$ & $5 / 7$ & $71 \%$ & $9 / 15$ & $60 \%$ \\
\hline & Tricuspid & $3 / 8$ & $38 \%$ & $2 / 7$ & $29 \%$ & $5 / 15$ & $33 \%$ \\
\hline & Pulmonary & $1 / 8$ & $13 \%$ & $0 / 7$ & $0 \%$ & $1 / 15$ & $7 \%$ \\
\hline \multicolumn{8}{|l|}{ BYPASS DATA } \\
\hline Total CPB duration (minutes) & Median (IQR) & 119.0 & $(97.0,151.0)$ & 124.0 & $(107.0,141.0)$ & 123.5 & $(99.0,146.0)$ \\
\hline
\end{tabular}

Details on missing data (Leukodepletion, Standard): $(1,0)$

$I Q R$ interquartile

${ }^{\mathrm{a}} 1$ patient in the leukodepletion filter group was missing CPB specific data, as this patient was an open and shut case 
Table 5 Post-operative primary and secondary outcomes

\begin{tabular}{|c|c|c|c|c|c|c|c|c|}
\hline & & \multicolumn{2}{|c|}{$\begin{array}{l}\text { Randomized to } \\
\text { leukodepletion filter } \\
(n=32)\end{array}$} & \multicolumn{2}{|c|}{$\begin{array}{l}\text { Randomized to } \\
\text { standard filter } \\
(n=31)\end{array}$} & \multicolumn{3}{|c|}{ Overall $(n=63)$} \\
\hline & & $\mathrm{n}$ & $\%$ & $\bar{n}$ & $\%$ & $\bar{n}$ & $\%$ & $(95 \% \mathrm{Cl})$ \\
\hline \multicolumn{9}{|l|}{ PRIMARY OUTCOME: } \\
\hline \multirow[t]{3}{*}{ Any AKI } & At any time & $14 / 32$ & $44 \%$ & $7 / 31$ & $23 \%$ & $21 / 63$ & $33 \%$ & $(0.22,0.46)$ \\
\hline & Prior to discharge & $14 / 29$ & $48 \%$ & $6 / 25$ & $24 \%$ & $20 / 54$ & $37 \%$ & \\
\hline & Between discharge and 6 weeks $~$ & $0 / 26$ & $0 \%$ & $1 / 24$ & $4 \%$ & $1 / 50$ & $2 \%$ & \\
\hline \multirow[t]{3}{*}{ Highest AKI stage } & Stage 1 & $9 / 14$ & $64 \%$ & $4 / 6$ & $67 \%$ & $13 / 20$ & $65 \%$ & \\
\hline & Stage 2 & $4 / 14$ & $29 \%$ & $1 / 6$ & $17 \%$ & $5 / 20$ & $25 \%$ & \\
\hline & Stage 3 & $1 / 14$ & $7 \%$ & $1 / 6$ & $17 \%$ & $2 / 20$ & $10 \%$ & \\
\hline \multirow[t]{3}{*}{ First instance of AKI } & Day 1 & $9 / 14$ & $64 \%$ & $2 / 6$ & $33 \%$ & $11 / 20$ & $55 \%$ & \\
\hline & Day 2 & $4 / 14$ & $29 \%$ & $3 / 6$ & $50 \%$ & $7 / 20$ & $35 \%$ & \\
\hline & Day 3 to discharge & $1 / 14$ & $7 \%$ & $1 / 6$ & $17 \%$ & $2 / 20$ & $10 \%$ & \\
\hline \multirow[t]{3}{*}{ Maximum AKI stage } & Day 1 & $6 / 14$ & $43 \%$ & $0 / 6$ & $0 \%$ & $6 / 20$ & $30 \%$ & \\
\hline & Day 2 & $7 / 14$ & $50 \%$ & $5 / 6$ & $83 \%$ & $12 / 20$ & $60 \%$ & \\
\hline & Day 3 to discharge & $1 / 14$ & $7 \%$ & $1 / 6$ & $17 \%$ & $2 / 20$ & $10 \%$ & \\
\hline \multicolumn{9}{|l|}{ SECONDARY OUTCOMES: } \\
\hline Need for haemodialysis & & $1 / 32$ & $3 \%$ & $0 / 31$ & $0 \%$ & $1 / 63$ & $2 \%$ & $(0.0,0.1)$ \\
\hline In hospital mortality & & $2 / 32$ & $6 \%$ & $0 / 31$ & $0 \%$ & $2 / 63$ & $3 \%$ & $(0.0,0.1)$ \\
\hline Infection complications & & $5 / 32$ & $16 \%$ & $4 / 31$ & $13 \%$ & $9 / 63$ & $14 \%$ & $(0.1,0.3)$ \\
\hline Length of post-operative stay & Median (IQR) days & 7.7 & $(5.8,13.9)$ & 8.8 & $(5.9,11.7)$ & 7.9 & $(5.9,12.0)$ & \\
\hline CICU length of stay & Median (IQR) days & 47.3 & $(24.8,100.0)$ & 48.5 & $(26.6,92.8)$ & 48.5 & $(25.0,98.3)$ & \\
\hline Ward length of stay & Median (IQR) days & 120.8 & $(94.0,168.8)$ & 141.5 & $(92.0,188.4)$ & 121.3 & $(93.5,187.0)$ & \\
\hline
\end{tabular}

\section{Length of post-op stay by AKI stage and group}
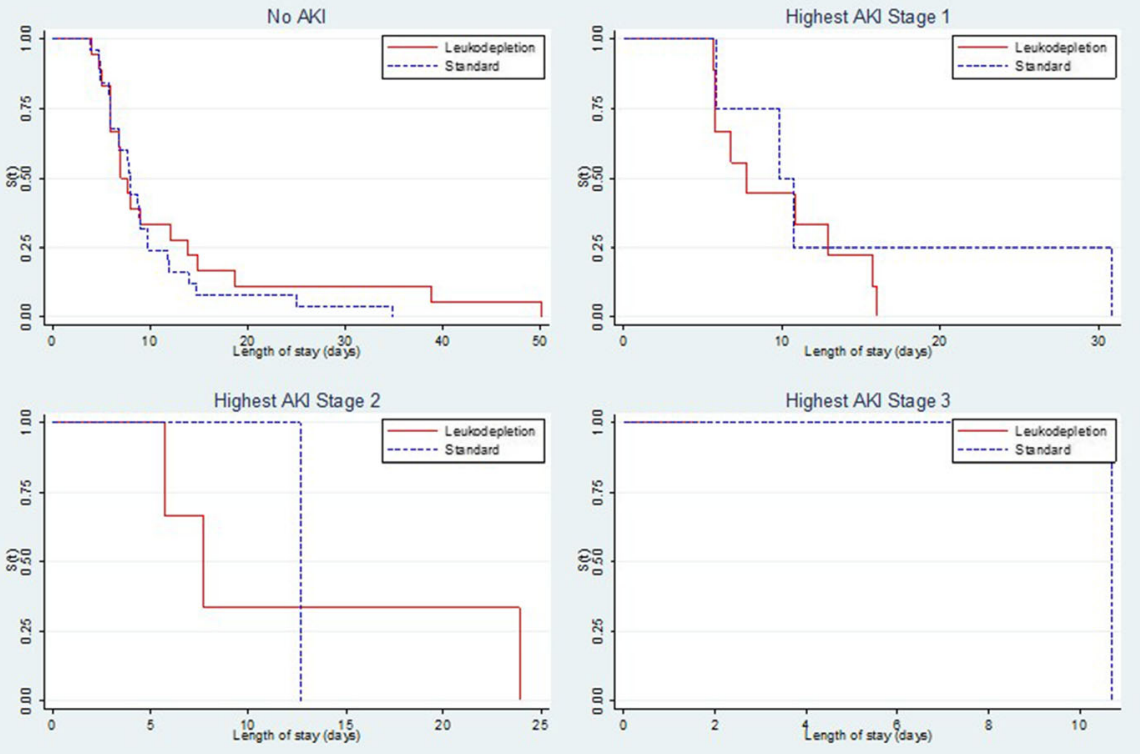

Fig. 2 Probability of remaining in hospital by AKI stage for each of leukodepletion versus the standard filter group. Kaplan-Meier plot showing no clear association between the incidence, severity of AKI and the secondary outcome of length of stay observed between the two groups and highest AKI stage 
patients in the leukodepletion filter group died during their post-operative stay. This was not directly related to the use of the filter. Infective complications occurred in 9/63 (14\%) patients, with a similar incidence in both groups (leukodepletion filter 16\%, standard filter 13\%).

Biomarker comparisons are summarized in Fig. 3.

The urinary RBP:Cr and KIM-1:Cr ratios were, on average, tended to be higher in the leukodepletion filter group compared to standard filter group (geometric mean ratio (GMR) $1.16,95 \%$ CI 0.80 to $1.69, p=0.45$ and $1.15,95 \% \mathrm{CI} 0.87$ to $1.52, p=0.35$ respectively). A proportion of samples were below the limit of detection for KIM-1:Cr, i.e. below $0.156 \mathrm{ng} / \mathrm{ml} \mathrm{(46/338} \mathrm{observa-}$ tions, $13.7 \%$ ). Urinary NGAL and Alb:Cr ratio were, on average, lower in the leukodepletion group (geometric mean ratio (GMR) $0.80,95 \%$ CI $0.54-1.18, p=0.29$ and $0.84,95 \%$ CI $0.51-1.39, p=0.53)$. None of the differences were statistically significant. Serum Cystatin C concentration was the only biomarker where the difference between the groups varied significantly with time $(p=0.011)$. After adjustment for pre-operative AKI severity, the data suggest a possible difference at $48 \mathrm{~h}$ (mean difference $0.11,95 \%$ CI 0.00 to $0.23, p=0.068$ ) with higher average values in the leukodepletion groups, but not at other times.

Comprehensive resource use data were collected. EQ$5 \mathrm{D}$ - $5 \mathrm{~L}$ scores were missing for $\leq 3(5 \%)$ patients at each time point (patients who died were assigned a score of zero for each time point after death). The mean (SD) EQ-5D single summary index scores for the cohort as a whole were $0.82(0.15)$ preoperatively, and $0.72(0.24)$, $0.78(0.22)$ and $0.85(0.22)$ at 5 days, 6 weeks and 12 weeks respectively, indicating that HRQoL improved with time after surgery. The MLHF scores showed a similar pattern.

The overall scores and the scores for both the physical and emotional subscales reduced from 5 days to 12 weeks indicating HRQoL improved over time (mean (SD)): overall score at baseline 37.4 (23.0), 5 days 45.6 (25.1), 6 weeks 29.6 (19.6), 12 weeks 16.2 (16.3). Resource use during follow up was captured for $36 / 38$ (95\%) participants at 6 weeks and 57/61 (93\%) participants at 3 months.

Overall, $3 \%$ of resource use data were missing: $1 \%$ for the in-patient period (which had the greatest number of resource use variables), $5 \%$ for follow up to 6 weeks, and $8 \%$ of follow up data to 3 months. On average, health related quality of life returned to pre-operative levels by 3 months.

A total of 203 adverse events (Supplementary material) were reported in 58 participants in the first 3 months after surgery, 66 of which were classified as serious adverse events (SAE). Forty SAEs were reported in 13 patients in the leucodepletion filter group compared to 26 SAEs in 13 patients in the standard filter group. Of the 203 adverse events reported, 182 were expected complications after cardiac surgery, i.e. they were listed in the

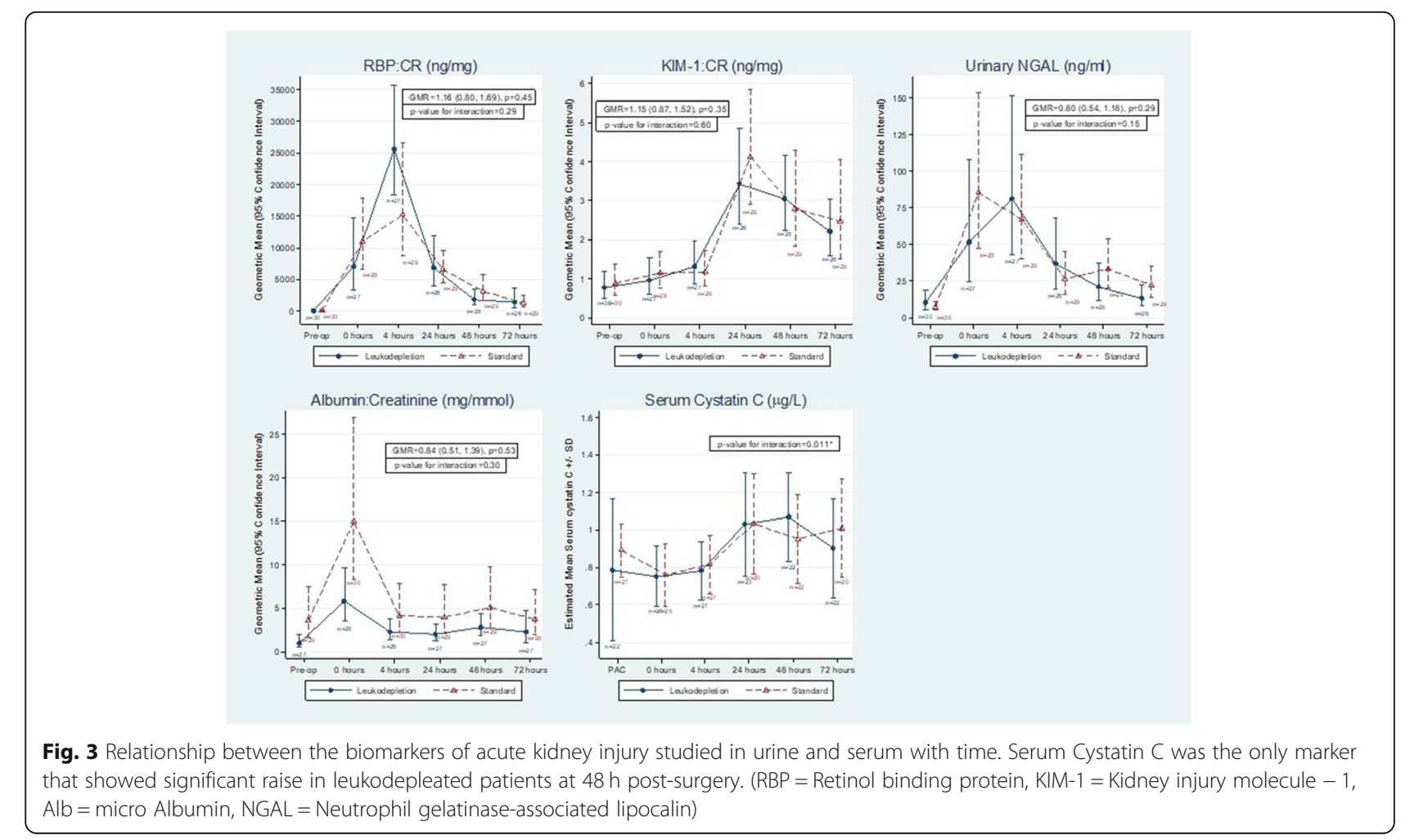


study protocol, and 21 were unexpected. Approximately one third of SAEs occurred after hospital discharge; SVT/AF requiring treatment was the most frequently reported SAE, which occurred in 5 participants (8\%). These differences however did not reach significance.

\section{Discussion}

Cochrane systematic review by the authors, failed to identify good quality trials in support of using leukodepletion in cardiac surgery in order to inform recommendations for a change in practice for its routine use [18]. Therefore this randomized trial was intended as a feasibility experiment to estimate key clinical, sub clinical and statistical parameters needed to design a larger more definitive trial in this subject.

However the results did not support the hypothesis that leukodepletion throughout cardiopulmonary bypass would reduce inflammatory response by removing activated leukocytes.

The incidence of the primary outcome of AKI was almost 1.5 times higher in the leukodepletion group compared to the standard. Serum levels of secondary outcome of Cystatin $\mathrm{C}$ showed evidence of subclinical AKI in the leukodepletion group as compared to the standard. However this did not translate into a difference in hospital stay or post procedural quality of life between the groups.

The study was conducted in a single centre so it may be more challenging to achieve similar compliance in a multi-centre setting. However, as was demonstrated in the Titre- 2 trial, with good engagement with study centers and participants excellent compliance with followup is achievable [30].

The incidence of AKI (the primary outcome) was 33\% which is consistent with previous reports [7], but notably higher than the $13.2 \%$ incidence reported in the Titre-2 trial which recruited 2000 cardiac surgery patients across 17 UK centers [31]. This could be explained by the differences in study cohort ( $41 \%$ of patients in Titre- 2 underwent isolated CABG surgery). In the subgroup of the Titre-2 cohort who underwent valve surgery (603 participants) the incidence was 13.8 and $4.3 \%$ were AKI stage 1 (personal communication).

In ROLO the primary outcome could not be calculated definitively for $9 / 63$ participants due to the Creatinine not being measured at the 6 week follow-up visit. This is a limitation, but with only one patient (2\%) developing AKI after discharge, not apparent immediately after surgery, it is unlikely that the cases with missing data would have experienced AKI.

Blinding of clinical teams to the intervention was successful and the treatment was delivered as planned, with only one cross-over for clinical reasons. There were 4 withdrawals after consent due to surgeon preference, reflecting the challenge of conducting a clinical trial in surgery.

Serum Cystatin C concentration was the only biomarker where the difference between the groups varied significantly with time indicative of renal insult at $48 \mathrm{~h}$. The anticipated 1.5 fold reduction in $\mathrm{RBP}: \mathrm{Cr}$ in the leukodepletion group [15] was not borne out in this study. Contrary to expectation the biomarker concentration was on average $16 \%$ higher in the leucodepletion filter group. The Alb:Cr ratio was lower in the leucodepletion group, but on average less than anticipated (GMR 0.84 against a target of 0.66). A significant proportion of KIM-1, samples were below the detection limit i.e. below $0.156 \mathrm{ng} / \mathrm{ml}$ (46/338 observations, $13.7 \%)$. However a sensitivity analysis omitting values below the limit of detection was consistent with the primary analysis.

Numerous factors contribute to the development of renal injury, including reductions in renal blood flow, actions of nephrotoxic drugs, injury to the proximal tubule epithelial cells, pro-inflammatory responses of renal endothelial cells, influx and activation of inflammatory leukocytes that further reduces renal blood flow through vascular congestion and promotes kidney injury [32, 33]. Although numerous studies have demonstrated the detrimental role of neutrophils, recent reports have uncovered a protective and possibly therapeutic role of neutrophils in AKI. Studies in animal models have revealed that neutrophils promote renal injury. In contrast, neutrophils are critical mediators of innate immunity. They respond rapidly (within minutes) to invading pathogens and to sites of tissue damage. Neutrophils clear invading pathogens by phagocytosis or by releasing toxic granules containing proteases and other enzymes and reactive oxygen species (ROS). For this reason, neutrophil depletion can lead to damage of host cells in the inflamed tissue. In several animal models, neutrophil accumulation in the injured kidney is a consistent and early finding and depletion of neutrophils or prevention of neutrophil tracking to the kidney reduces kidney injury. However, these results may be related to differences in experimental models and limitations to methods for neutrophil depletion. In addition neutrophils releasing pro-inflammatory cytokines, interferon IFN- $\gamma$ and interleukin IL-17, and the chemokine CXCL1, in the injured kidney [34-36] and direct or indirect release of antiinflammatory cytokines IL- 6 IL-10, etc. may suggest a more complex balance of pro and anti-inflammatory mechanism of neutrophils related pathogenesis of kidney injury.

\section{Conclusion}

The ROLO trial has demonstrated that a higher incidence of AKI and raised biomarkers of renal injury are associated with the use of a leukodepletion filter. 
Addition of a leukodepletion filter to the bypass circuit for valvular heart surgery cannot be supported for the purpose of limiting post-operative renal dysfunction. Other interventions to reduce the incidence of this important complication should be explored.

\section{Abbreviations}

AE: Adverse event - any undesirable event in a subject receiving treatment according to the protocolincluding occurrences which are not necessarily caused by or related to administration of the research procedures; AF: Atrial Fibrillation; AKI: Acute kidney injury - an acute increase in serum creatinine > $26.4 \mu \mathrm{mol} / \mathrm{l}$ or a percentage increase in serum creatinine of more than or equal to 50\%; AR: Adverse reaction - any undesirable experience that has happened a subject while taking a drug that is suspected to be caused by the drug or drugs; ARDS: Acute respiratory distress syndrome; ARF: Acute renal failure; AVR: Aortic valve replacement/repair; BHI: Bristol Heart Institute; BRI: Bristol Royal Infirmary; BRU: Biomedical Research Unit; CABG: Coronary artery bypass grafting; CAD: Coronary artery disease; CKD Stage: International classification of chronic kidney disease; CPB: Cardiopulmonary bypass - a technique that temporarily takes over the function of the heart and lungs during surgery, maintaining the circulation of blood and the oxygen content of the body whilst allowing surgeons to access static cardiac tissue; CSAAKI: Cardiac surgery associated acute kidney injury; CTEU: Clinical Trials and Evaluation Unit; CWH: Continuous veno-venous hemofiltration; DM: Diabetes mellitus; DMSC: Data monitoring and safety committee; ECG: Graphical representation of electrical activity of the heart over time, as recorded by an electrocardiograph; eGFR: Estimated glomerular filtration rate: derived from gender, age, ethnicity and serum creatinine; ELISA: Enzyme-linked immunosorbent assay; ESRF: End stage renal disease requiring dialysis, CKD stage 5; HDU HRQoL: High dependency unit, Health-related quality of life: ICH-GCP: International conference for harmonisation of good clinical practice; ICU: Intensive care unit; IL-: Interleukin- a validated specific marker of acute kidney injury; ITT: Intension to treat; KDIGO: Kidney Disease Improving Global Outcomes; KIM-1: Kidney Injury Molecule-1; LDH: Lactate dehydrogenase - an enzyme often used as a marker of tissue breakdown; high levels of LDH indicate tissue damage; LFT: Liver function test; MACE: Major cardiac adverse event; MI: Myocardial infarction; MLHF: Minnesota living with heart failure; MRC: Medical Research Council; NCEPOD: National Confidential Enquiry into Patient Outcome and Death: NGAL: Neutrophil gelatinase associated lipocalin - a specific marker of acute kidney injury; NIHR: National Institute for Health Research; RCT: Randomised controlled trial; RIFLE: Risk, Injury, Failure, Loss of kidney function; REC: Research ethics committee; SAE: Serious adverse event - events which result in death, are life threatening, require hospitalisation or prolongation of hospitalisation, result in persistent or significant disability or incapacity; SAR: Serious adverse reaction; SIRS: ${ }^{\text {Sy }}$ stemic inflammatory response syndrome; SOP: Standard operating procedure; SSAR: Suspected serious adverse reaction; SUSAR: Suspected unexpected serious adverse reaction - an untoward medical occurrence suspected to be related to a medicinal product that is not consistent with the applicable product information and is serious; SVT: Supra ventricular tachycardia; TIA: Transient ischemic attack; TMG: Trial management group; TSC: Trial steering committee; UH Bristol: University Hospitals Bristol NHS Foundation Trust

\section{Supplementary Information}

The online version contains supplementary material available at https://doi. org/10.1186/s13019-021-01402-4.

\section{Additional file 1.}

\section{Acknowledgements}

Thanks to Michelle Stephens research and development manager, Helen Spickett research performance manager and Mandy Dixon research grant coordinator at Blackpool and Lancashire University. The trial was sponsored and carried out at Blackpool, Fylde and Wyre Hospitals NHS Foundation Trust Research \& Development department. The trial was registered by the International Standard Randomized Controlled Trial Number Registry ISRC TN42121335 (http://www.isrctn.com/ISRCTN42121335).

\section{Authors' contributions}

All authors contributed equally to this research.

\section{Funding}

This trial was financially supported by the National Institute of Health

Research (Research for Patient Benefit), award ID: PB-PG-0711-25090.

\section{Availability of data and materials}

The trial was run by Bristol clinical trials and evaluation unit through whom all supporting data could be made available.

\section{Declarations}

\section{Ethics approval and consent to participate}

Research ethics approval was given by the National Research Ethics Service (NRES) Committee, North West - Preston (Ref. 13/NW/0728) which conforms to the provisions of the Declaration of Helsinki. All participants gave written informed consent to participate in this trial.

\section{Consent for publication}

This manuscript does not contain individual person's data in any form.

\section{Competing interests}

The authors have no competing or financial interest in the information contained in the manuscript.

\section{Author details}

${ }^{1}$ Department of Cardiothoracic Surgery, Freeman Hospital, High Heaton, Newcastle upon Tyne NE7 7DN, UK. ${ }^{2}$ School of Health and Medicine, Lancaster University, Bailing, Upper Market Street, Lancaster, Lancashire LA1 4YW, UK. ${ }^{3}$ Renal Unit, Lancashire Teaching Hospitals NHS Foundation Trust, Sharoe Green Lane, Fulwood, Preston, Lancashire PR2 9HT, UK. ${ }^{4}$ Department of Cardiothoracic Surgery, Blackpool Victoria Hospital, Blackpool FY3 8NR, UK. ${ }^{5}$ Department of Public Health, University of Oxford, Rosemary Rue Building, Old Road Campus, Headington, Oxford OX3 7LF, UK. ${ }^{6}$ Clinical Trials and Evaluation Unit, Bristol Medical School, University of Bristol, Bristol BS2 8HW, UK.

Received: 10 July 2020 Accepted: 8 March 2021

Published online: 26 March 2021

\section{References}

1. D'Onofrio A, Cruz D, Bolgan I, Auriemma S, et al. RIFLE criteria for cardiac surgery-associated acute kidney injury: risk factors and outcomes. Congest Heart Fail. 2010;1:S32-6.

2. Vives M, Monedero P, Perez-Valdivieso JR, Garcia-Fernandez N, et al. External validation and comparison of three scores to predict renal replacement therapy after cardiac surgery: a multicenter cohort. Int J Artif Organs. 2011; 34:329-38.

3. Ristikankare A, Poyhia R, Kuitunen A, Skrifvars M, et al. Serum cystatin C in elderly cardiac surgery patients. Ann Thorac Surg. 2010;89:689-94.

4. Grayson AD, Khater M, Jackson M, Fox MA. Valvular heart operation is an independent risk factor for acute renal failure. Ann Thorac Surg. 2003;75: 1829-35.

5. Sixth National Adult Cardiac Surgical Database Report: Demonstrating Quality. The society for cardiothoracic surgery in Great Britain \& Ireland 2008.

6. Dasta JF, Kane-Gill SL, Durtschi AJ, Pathak DS, Kellum JA. Costs and outcomes of acute kidney injury (AKI) following cardiac surgery. Nephrol Dial Transplant. 2008;23:1970-4.

7. Stewart JFG, Smith N, Kelly K, Mason M. Adding insult to injury: a review of the care of patients who died in hospital with a primary diagnosis of acute kidney injury. London: National Confidential Enquiry into Patient Outcome and Death (NCEPOD); 2009.

8. Mangano CM, Diamondstone LS, Ramsay JG, Aggarwal A, et al. Renal dysfunction after myocardial revascularization: risk factors, adverse outcomes, and hospital resource utilization. The Multicenter Study of Perioperative Ischemia Research Group. Ann Intern Med. 1998:128:194-203.

9. Brown JR, Kramer RS, Coca SG, Parikh CR. Duration of acute kidney injury impacts long-term survival after cardiac surgery. Ann Thorac Surg. 2010;90: 1142-8. 
10. Landis RC, Brown JR, Fitzgerald D, Likosky DS, et al. Attenuating the systemic inflammatory response to adult cardiopulmonary bypass: a critical review of the evidence base. J Extra Corpor Technol. 2014;46:197-211.

11. Day JRS, Taylor KM. The systemic inflammatory response syndrome and cardiopulmonary bypass. Int J Surg. 2005;3:129-40.

12. Warltier DC, Laffey JG, Boylan JF, Cheng DCH. The systemic inflammatory response to cardiac surgery: implications for the anesthesiologist. Anesthesiology. 2002;97:215-52.

13. Kumar AB, Suneja M. Cardiopulmonary bypass-associated acute kidney injury. Anesthesiology. 2011;114:964-70.

14. Alexiou C, Sheppard S, Tang A, Rengarajan A, et al. Leukocytes-depleting filters preferentially remove activated leukocytes and reduce the expression of surface adhesion molecules during the simulated extracorporeal circulation of human blood. ASAIO J. 2006;52:438-44.

15. Tang AT, Alexiou C, Hsu J, Sheppard SV, et al. Leukodepletion reduces renal injury in coronary revascularization: a prospective randomized study. Ann Thorac Surg. 2002;74:372-7.

16. Charuhas V, Thakar CV, Arrigain S, Worley S, Yared JP. A clinical score to predict acute renal failure after cardiac surgery. J Am Soc Nephrol. 2005;16: 162-8.

17. Bolcal C, Akay HT, Bingol H, Doganci S, et al. Leukodepletion improves renal function in patients with renal dysfunction undergoing on-pump coronary bypass surgery: a prospective randomized study. Thorac Cardiovasc Surg. 2007:55:89-93.

18. Spencer S, Tang A, Khoshbin E. Leukodepletion for patients undergoing heart valve surgery. Cochrane Database Syst Rev. 2013;31:1465-858.

19. Tang AT, El-Gamel A, Keevil B, Yonan N, Deiraniya AK. The effect of 'renaldose' dopamine on renal tubular function following cardiac surgery: assessed by measuring retinol binding protein (RBP). Eur J Cardiothorac Surg. 1999;15:717-21.

20. Zheng M, Shi M, Pei Z, et al. The transcription factor RBP-J is essential for retinal cell differentiation and lamination. Mol Brain. 2009;2:38.

21. Koyner JL, Vaidya VS, Bennett MR, Ma Q, et al. Urinary biomarkers in the clinical prognosis and early detection of acute kidney injury. Clin J Am Soc Nephrol. 2010;5:2154-65.

22. el-Gamel A, Yonan N, Keevil B, Nicholls S, et al. Measurement of microalbumin and retinol binding protein in cardiac and lung transplant recipients. Transplant Proc. 1995;27:1969-72.

23. Peralta CA, Katz R, Sarnak MJ, Ix J, et al. Cystatin C identifies chronic kidney disease patients at higher risk for complications. J Am Soc Nephrol. 2011;22: 147-55

24. Wagener $\mathrm{G}$, Jan M, Kim M, Mori K, et al. Association between increases in urinary neutrophil gelatinase-associated lipocalin and acute renal dysfunction after adult cardiac surgery. Anesthesiology. 2006;105:485-91.

25. Rector T, Kubo S, Cohn J. Patients' self-assessment of their congestive heart failure. Part 2: content, reliability and validity of a new measure, the Minnesota living with heart failure questionnaire. Heart Fail. 1987;3:198-209.

26. EuroQol Group. EuroQol--a new facility for the measurement of healthrelated quality of life. Health Policy. 1990;16:199-208.

27. Brooks R. EuroQol: the current state of play. Health Policy. 1996:37:53-72.

28. Lewington AKS. Clinical practice guidelines - acute kidney injury. UK Ren Assoc. 2009.

29. Spencer S, Tang A, Khoshbin E. Leukodepletion for patients undergoing heart valve surgery (protocol). Cochrane Database Syst Rev. 2011;12.

30. Reeves BC, Pike K, Rogers CA, Brierley RC, et al. A multicentre randomized controlled trial of transfusion indication threshold reduction on transfusion rates, morbidity and health-care resource use following cardiac surgery (TITRe2). Health Technol Assess. 2016;20:1-260.

31. Murphy GJ, Pike K, Rogers CA, Wordsworth S, et al. Liberal or restrictive transfusion after cardiac surgery. N Engl J Med. 2015;372:997-1008.

32. Kinsey GR, Okusa MD. Pathogenesis of acute kidney injury: foundation for clinical practice. Am J Kidney Dis. 2011;58:291-301.

33. Schrier RW, Wang W, Poole B, Mitra A. Acute renal failure: defi nitions, diagnosis, pathogenesis, and therapy. J Clin Invest. 2004;114:5-14.

34. Li L, Huang L. Sung SS. NKT cell activation mediates neutrophil IFN-gamma production and renal ischemia-reperfusion injury. J Immunol. 2007;178: 5899-911.

35. Li L, Huang L, Vergis AL. IL-17 produced by neutrophils regulates IFNgamma-mediated neutrophil migration in mouse kidney ischemiareperfusion injury. J Clin Invest. 2010;120:331-42.
36. Tadagavadi RK, Reeves WB. Renal dendritic cells ameliorate nephrotoxic acute kidney injury. J Am Soc Nephrol. 2010;21:53-63.

\section{Publisher's Note}

Springer Nature remains neutral with regard to jurisdictional claims in published maps and institutional affiliations.
Ready to submit your research? Choose BMC and benefit from:

- fast, convenient online submission

- thorough peer review by experienced researchers in your field

- rapid publication on acceptance

- support for research data, including large and complex data types

- gold Open Access which fosters wider collaboration and increased citations

- maximum visibility for your research: over $100 \mathrm{M}$ website views per year

At $\mathrm{BMC}$, research is always in progress.

Learn more biomedcentral.com/submissions 